\title{
Association of Blood Biomarkers and Autoimmunity with Immune Related Adverse Events in Patients with Cancer treated with Immune Checkpoint Inhibitors
}

\section{Despina Michailidou}

Division of Rheumatology, Dept. of Medicine, University of Washington, Seattle, WA

\section{Ali Khaki}

Division of Medical Oncology, Dept. of Medicine, University of Washington; Fred Hutchinson Cancer Research Center; Seattle Cancer Care Alliance, Seattle, WA

\section{Maria Morelli}

Gastrointestinal Medical Oncology, The University of Texas, MD Anderson Comprehensive Cancer Center, Houston, Texas

\section{Leonidas Diamantopoulos}

Dept. of Medicine, University of Pittsburgh, Pittsburgh, PA

\section{Namrata Singh}

Division of Rheumatology, Dept. of Medicine, University of Washington, Seattle, WA

\section{Petros Grivas ( $\nabla$ pgrivas@uw.edu )}

Division of Medical Oncology, Dept. of Medicine, University of Washington; Fred Hutchinson Cancer Research Center; Seattle Cancer Care Alliance, Seattle, WA

\section{Research Article}

Keywords: cancer, immune checkpoint inhibitors (ICls), immune related adverse events (irAEs),overall survival (OS)

Posted Date: January 4th, 2021

DOl: https://doi.org/10.21203/rs.3.rs-136849/v1

License: (c) (i) This work is licensed under a Creative Commons Attribution 4.0 International License. Read Full License

Version of Record: A version of this preprint was published at Scientific Reports on April 27th, 2021. See the published version at https://doi.org/10.1038/s41598-021-88307-3. 


\section{Abstract}

Background: Patients with cancer treated with immune checkpoint inhibitors (ICls) develop immune related adverse events (irAEs), however biomarkers are lacking. We hypothesized that clinicopathologic and laboratory factors would be associated with irAE risk and overall survival (OS) in this population.

Methods: In a retrospective study of patients treated with ICls we collected clinicopathologic, laboratory, irAEs and outcomes data. The association between baseline blood biomarkers, clinicopathologic features and irAEs was assessed by logistic regression adjusting for age, sex, smoking, cancer type, performance status, concomitant other systemic therapy, history of autoimmune disease (AD) and chronic infection. Optimal cutoff values of biomarkers were identified by recursive partitioning analysis.

Results: 470 patients were identified; 156 (33\%) developed irAEs, which were associated with baseline absolute lymphocyte count $>2.6 \mathrm{k} / \mathrm{ul}$ (adjusted [a]OR:4.12), neutrophil to lymphocyte ratio (NLR) $\leq 5.3$ (aOR:2.08) and monocyte to lymphocyte ratio (MLR) $\leq 0.73$ (aOR:3.11). Patients with pre-existing $A D$ (aOR:2.81), family history of $A D$ (aOR:5.86), and ICl combination (aOR:2.26) had higher odds of irAEs. Baseline NLR $\leq 5.3$ (aHR:0.68) and MLR $\leq 0.73$ (aHR:0.43) were associated with longer OS.

Conclusion: irAE were associated with autoimmune history, ICl combination and baseline laboratory measurements. Lower NLR and MLR may have favorable prognostic value. Our hypothesis-generating findings require validation in larger prospective studies.

\section{Introduction}

Immune checkpoint inhibitors (ICls) have altered the therapeutic landscape of immunotherapy in cancer (1). ICls block regulatory T-cells immunosuppression by blocking intrinsic down-regulators of immunity, such as cytotoxic T-lymphocyte antigen 4 (CTLA-4) and programmed cell death 1 (PD-1) or its ligand, programmed cell death ligand 1 (PD-L1) (2). This inhibition can lead to an effective anti-tumor immune response but can trigger significant immune related adverse events (irAEs) causing significant morbidity, mortality and increasing costs (3).

Target sites of irAEs can be any human tissue including, but not limited to, gut, skin, lungs, and endocrine glands (4). Rheumatologic irAEs have been reported $(5,6)$ and require the collaborative, inter-disciplinary management by rheumatologists and oncologists. There can be hesitation to administer ICls to patients with pre-existing autoimmune disease (AD), as $20-40 \%$ of them develop unrelated irAEs or exacerbation of their underlying disease $(1,7,8)$. Thus, there is an urgent unmet need to identify additional demographic, clinicopathologic and laboratory features that may be associated with higher irAE risk, helping oncologists and patients carefully weigh the benefit/risk ratio of ICls in practice.

The objectives for this retrospective cohort study of patients who received ICls for either solid tumors or hematological malignancies were to: (1) explore whether there is an association between irAEs and a. absolute blood cell counts and their ratios at baseline; b. select clinicopathologic features; (2) to evaluate 
the potential association between baseline blood count biomarkers and overall survival (OS). We hypothesized that baseline blood counts and select clinicopathologic features would be associated with the risk of irAEs and influence OS of patients with cancer treated with ICls.

\section{Methods}

\section{Study population}

Patients were included from an IRB-approved retrospective observational cohort study at the University of Washington and Seattle Cancer Care Alliance (SCCA) in Seattle, WA (IRB ID: STUDY00008393). Patients who initiated therapy with anti-PD-1 (nivolumab, pembrolizumab, cemiplimab), anti-PD-L1 (atezolizumab, durvalumab, avelumab), or anti-CTLA-4 (ipilimumab, tremelimumab) ICls between January 2018 and October 2018 were included in the study. We used analytical search algorithms to identify all consecutive patients with new $\mathrm{ICl}$ initiation during this time period.

\section{Patient and public involvement}

The authors declare no direct patient and public involvement in study design.

\section{Clinical and laboratory assessment}

Data was abstracted using REDCap data collection tools hosted by the Institute of Translational Health Sciences (ITHS) (9). Data abstracted included demographics, any personal and/or family history of AD, personal history of chronic infection, smoking history, laboratory values, Eastern Cooperative Oncology Group performance status (ECOG PS), sites of metastatic disease at time of ICI initiation, whether concomitant administration of ICls or other systemic therapy was given, date of irAE development, site/organ of irAE, vital status, and date of death or last follow up. Diagnosis of irAE was defined as new onset adverse event that patients developed after ICI initiation and was determined by the treating provider to be attributed to $\mathrm{ICl}$ in the absence of other etiologies based on consistent chart review. Only clinically significant irAEs that required pharmacological intervention and/or hospitalization were recorded. Chronic infection was defined as history of human immunodeficiency virus (HIV), hepatitis $C$ $(\mathrm{HCV})$, hepatitis $B(\mathrm{HBV})$, or tuberculosis. Baseline laboratory parameters recorded included absolute neutrophil count (ANC), absolute lymphocyte count (ALC), absolute monocyte count (AMC), and platelet count (PLT). Neutrophil to lymphocyte ratio (NLR), monocyte to lymphocyte ratio (MLR) and platelet to lymphocyte ratio (PLR) were also calculated.

\section{Statistical analysis}


We independently evaluated whether baseline blood counts, personal or family history of AD, treatment with combination $\mathrm{ICl}$ or history of chronic infection were associated with irAE development using multivariable logistic regression. For the laboratory analyses, we a priori adjusted for age, sex, smoking history, cancer type (hematological vs solid malignancy), ECOG PS, concomitant other systemic therapy, personal or family history of $A D$, and personal history of chronic infection. For the non-laboratory analyses, we a priori adjusted for age, sex, smoking history, cancer type (hematological vs solid malignancy), ECOG PS, and concomitant systemic therapy. The results were reported using odds ratio (OR) with $95 \%$ confidence interval $(\mathrm{Cl})$.

In addition to testing the lab values on a continuous scale, we used the recursive partitioning analysis (RPA) to identify the optimal cut-off values that may be associated with irAE development for absolute cell counts and ratios. Once identified, we evaluated the association between these cut-off values and irAE development using similar multivariable models as stated above.

We also performed an exploratory analysis evaluating the potential association between our exposures of interest (baseline laboratory values, personal or family history of $A D$, personal history of chronic infection and concomitant $\mathrm{ICI}$ ) and overall survival (OS). OS was calculated from the first day of treatment with $\mathrm{ICI}$ to death of any cause. Patients who did not die were censored at the date of last follow-up. We used the Kaplan-Meier method to estimate OS, and multivariable Cox regression to compare OS. We tested lab values using the continuous scale for the OS analysis; we also tested the cut-off points derived based on irAE development for any laboratory value that had a significant association with irAE. Hazard ratios (HR) with $95 \% \mathrm{Cl}$ were reported. For all analyses, $p$ values $<0.05$ were considered statistically significant. Data analysis was carried out using STATA (v16.1) and R software (3.5.0).

\section{Results}

\section{Demographic characteristics}

A total of 470 patients were included in our study with median age 65. Patients were predominantly nonHispanic white (87\%) and men (59\%). Baseline demographic features are shown in Table 1; 315 patients $(67 \%)$ received monotherapy with anti-PD-1, 56 (12\%) with anti-PD-L1, only $2(<1 \%)$ received anti-CTLA-4 monotherapy, and, 88 (19\%) received concomitant ICls.

\section{Types and frequency of irAEs}

Overall, 156 out of 470 patients (33\%) developed irAEs. A total of 212 irAEs were recorded as 42 out of 470 (9\%) of patients developed >1 irAEs; (15\% rheumatologic; $85 \%$ non-rheumatologic). The occurrence of different irAE categories is shown in Table 2. The median number of $\mathrm{ICl}$ doses before the onset of irAE was four (range 1-29). The most common rheumatologic irAE was inflammatory arthritis (IA) (11/31; $35 \%)$ and the most common non-rheumatologic irAE was hypothyroidism $(43 / 181 ; 24 \%)$. 


\section{Association of blood count biomarkers with irAEs}

Baseline ANC, ALC, AMC and PLT were available for 441 (94\%), 391 (83\%), 391 (83\%) and 459 (98\%) out of 470 patients, respectively. Higher baseline ALC modeled as a continuous variable was the only absolute cell count that was associated with higher odds of irAEs (adjusted [a]OR: 1.44, 95\%Cl: 1.06-1.95, $p=0.02$, Table $3 A$ ). In addition, baseline ALC $>2.6 \mathrm{k} / \mathrm{ul}$ was associated with higher odds of irAE occurrence (aOR: 4.12, 95\%Cl: 1.64-10.42, $\mathrm{p}=0.003$; Table 3B), indicating that patients with cancer and baseline lymphocytosis may have a greater likelihood of developing irAEs.

Among 470 patients treated with ICls, 390 (83\%) had baseline complete blood counts available for NLR, MLR and PLR calculation. Higher baseline MLR as a continuous variable (aOR: $0.34,95 \% \mathrm{Cl}: 0.17-0.70$, $p=0.003$ ) was associated with lower odds of irAE development (Table $3 A$ ). In addition, baseline NLR $\leq 5.3$ (aOR: $2.08,95 \% \mathrm{Cl}: 1.20-3.58, \mathrm{p}=0.01$ ) and MLR $\leq 0.73$ (aOR: 3.11, 95\% Cl: 1.65-5.89, $\mathrm{p}<0.001$ ) were both associated with higher odds of irAE (Table 3B).

\section{Association of clinicopathologic features with irAEs}

Personal or family history of $A D$, history of chronic infection, and combination ICl were investigated among all 470 patients. Both personal (aOR: $2.81,95 \% \mathrm{Cl}: 1.64-4.79, \mathrm{p}<0.001$ ) and family history of $A D$ (aOR: 5.86, 95\%Cl:2.21-15.53, p<0.001) were significantly associated with irAEs. In addition, combination of ICls (aOR: $2.26,95 \% \mathrm{Cl}: 1.35-3.77, p=0.002$ ) was also associated with higher odds of irAEs, while history of chronic infection (aOR: $0.42,95 \% \mathrm{Cl}: 0.19-0.92, p=0.03$ ) was associated with lower odds of irAEs.

\section{Association of blood count biomarkers with OS}

In an exploratory analysis, we evaluated the association between baseline absolute cell counts (and the cut-off points derived using RPA in association to irAE development) and OS. Multivariable Cox regression analysis showed that higher baseline AMC level and higher baseline MLR level as continuous variables, were both significantly associated with shorter OS (aHR: 2.04, 95\% Cl: $1.18-3.50, p=0.01$, and aHR: $1.27,95 \% \mathrm{Cl}: 1.05-1.54, \mathrm{p}=0.02$, respectively, Table $3 \mathrm{~A}$ ). We further tested whether the laboratory cutpoints associated with irAEs were also associated with OS. There was no significant association between baseline ALC >2.6k/ul and OS (aHR: 0.61, 95\%Cl: 0.28-1.35, p=0.22; Figure 1A). However, baseline NLR $\leq 5.3$ and MLR $\leq 0.73$ were associated with higher OS (aHR: $0.68,95 \% \mathrm{Cl}: 0.48-0.95, p=0.02$; aHR: $0.43,95 \% \mathrm{Cl}: 0.31-0.61, p<0.001$, respectively, Figure 1B, 1C).

\section{Association of clinicopathologic features with OS}


Patients with personal or family history of $A D$ receiving $\mathrm{ICl}$ did not have significantly different $\mathrm{OS}$ compared to those without such history (aHR: 1.24, 95\% Cl: $0.86-1.79, \mathrm{p}=0.24$; aHR: $0.65,95 \% \mathrm{Cl}: 0.27$ $1.60, p=0.35$, respectively). Similarly, neither the concomitant combination of ICls nor the history of chronic infection showed a significant association with OS (aHR: 1.23, 95\% Cl: $0.86-1.78, p=0.25$; aHR: 1.20 95\%Cl: $0.76-1.88, p=0.43$, respectively).

\section{Discussion}

Utilizing comprehensive clinicopathologic and laboratory data, this retrospective cohort study details the relationships between specific blood count biomarkers, as well as patient's health history with the development of irAEs. In our study, development of irAEs was significantly associated with higher baseline ALC (optimal cut-off $>2.6 \mathrm{k} / \mathrm{ul}$ ), lower baseline NLR (optimal cut-off $\leq 5.3$ ) and lower baseline MLR (optimal cut-off $\leq 0.73$ ). Additionally, use of concomitant ICl, family and personal history of autoimmune diseases were identified as independent factors associated with irAEs. Notably, while family history has been assumed or implicated as an independent risk factor, our study is one of the first to show this association in a relatively large cohort.

Regarding blood cell count subsets, our findings partially align with prior studies that demonstrated that patients with solid tumors treated with PD-1 inhibitors (nivolumab or pembrolizumab) who had an ALC $>2$ $\mathrm{k} / \mathrm{ul}$ at baseline were at a higher risk of irAEs (10). Our results demonstrated that ALC $>2.6 \mathrm{k} / \mathrm{ul}$ at the time of initiation of $\mathrm{ICl}$ was associated with higher odds for irAE development. This finding may suggest that peripheral lymphocytosis may reflect a state of $T$ cell activation that may result not only in enhancement of anti-tumor activity but also higher risk of toxicity. If our hypothesis is validated by future studies, exploration of lymphocyte-directed therapies may be a clinically relevant hypothesis for the management of irAEs that can be explored in clinical trials $(10,11)$.

Multiple prior studies have also shown the association between NLR, irAE development and OS (12-14). In a study of patients with advanced non-small cell lung cancer (NSCLC) treated with anti-PD-(L) 1 agents, baseline NLR $<3$ was significantly associated with development of irAEs. Another study of patients with NSCLC treated with ICls also noted an association between NLR $<5$ and irAEs. In addition, a different cohort of patients with NSCLC treated with nivolumab showed that pretreatment NLR $\geq 5$ was associated with inferior OS. Similar to those findings, we demonstrated that pretreatment NLR $\leq 5.3$ was associated with higher odds of irAEs and longer OS. These results suggest that lower NLR might enable a more efficient anti-tumor immune response but also portend a higher risk of immune-related toxicity. This may be due to pro-inflammatory cytokines in the plasma of patients with higher NLR, which may impact the tumor microenvironment and facilitate aggressive tumor behavior (15). In addition, higher NLR has been associated with an increase in peripheral blood regulatory $T$ cells that can suppress excessive immune responses (16).

Increased MLR is another potential indicator of systemic inflammation that has been associated with shorter OS in patients with cancer treated with immunotherapy (17). In our study, we showed that higher 
MLR and AMC were associated with shorter OS. Type II tumor-associated macrophages from circulating monocytes can suppress the immune reaction against tumor cells $(18,19)$. Lower $A M C$ as a continuous variable during ipilimumab treatment in patients with melanoma was associated with favorable outcome (20). In our study, baseline MLR $\leq 0.73$ was associated with almost three-fold higher odds of irAEs and also longer OS. Lower MLR ratio may possibly reflect the balance between the favorable role of lymphocytes and unfavorable effect of particular monocyte types in this setting.

In addition to laboratory values, demographic and clinicopathologic features, such as family and/or personal history of $A D$, may be relevant to the development of IRAEs. There are several reports of familial aggregation of rheumatoid arthritis and/or other autoimmune diseases, including systemic lupus erythematosus, Sjögren's syndrome, ankylosing spondylitis, systemic sclerosis, polymyalgia rheumatica, Hashimoto thyroiditis, hypothyroidism, psoriasis, vasculitis or sarcoidosis. (21-24). The presence of diverse autoimmune disorders in family members of patients who developed irAEs may imply potential hereditary susceptibility to autoimmunity. Therefore, genetic factors may possibly play a role in irAE development, while detailed family history of $A D$ may be a useful tool in identifying those who may be at higher risk. We did not perform additional analyses regarding the specific types, severity, or the degree of relatives with $A D$, but this can be the focus of future dedicated studies.

Many studies have shown an increased risk of irAE or AD flare among patients with pre-existing $A D$ or prior irAE (25-27). In a recent systematic review that studied the use of ICls in the treatment of patients with cancer and pre-existing AD, exacerbation of pre-existing AD (41\%), de novo irAEs (25\%), or both (9\%) developed in $75 \%$ of patients (28). Initially, patients with pre-existing AD were excluded from many clinical trials of ICls because of increased risk of development of irAEs (29). However, patients with well controlled, mild to moderate pre-existing AD may be often treated with ICls. For patients with metastatic melanoma or NSCLC also with AD requiring no or only low dose immunosuppression, data from retrospective studies suggest that a minority of patients (20\%-40\%) experienced exacerbation of their $A D$ after administration of ICls, which were relatively manageable (30-32). In our study, $17 \%$ of patients had a pre-existing history of $A D$ and only $3 \%$ of patients in our cohort were on immunosuppressive therapy (including steroids) prior to ICl initiation of (Table 1). The increased irAE rate with the use of concomitant ICls is well described in the literature $(1,33,34)$.

Our study has a number of limitations inherent to the retrospective study nature. First, patients received single agent and/or combination of ICls for different cancers, indicating major heterogeneity and variability in the patient population, clinical setting, functional status, medical comorbidities, organ function, treatment duration, prior therapies, surveillance/monitoring strategies and follow up times. Although our statistical analysis attempted to adjust for differences in several clinicopathologic factors, recall, selection and residual confounding biases are inevitable; therefore, association may not reflect causation. There is also a possibility of misclassification related to data availability and missing/unknown data. For example, charts may not have the most complete family histories of AD so this exposure may be under-represented; we also did not capture irAE grade. Additionally, we acknowledge that this is a single center study amenable to potential regional influences. 
The study has also strengths. Our sample size of 470 diverse patients with a wide variety of tumor types and the systematic strategy for granular data abstraction enabled a solid hypothesis-generating identification of putative factors associated with irAEs. A significant proportion of our results is consistent with previously published data and suggest that blood count subsets could possibly be used in the future, upon further validation, as inexpensive and easily obtained putative biomarkers to help predict the development of irAEs.

To conclude, our findings in this retrospective cohort study support the notion that in patients with cancer prior to initiation of $\mathrm{ICl}$, family or personal history of autoimmune disease, as well as baseline laboratory assessment are significantly associated with IRAEs. Upon further validation, our findings may possibly contribute to more accurate estimation of irAEs and support discussions on future development of prognostic nomograms and models that can be further tested in clinical trials.

\section{Declarations}

\section{Acknowledgements:}

We would like to acknowledge Dr. Eshana Shah for her contributions in preparing the IRB protocol.

\section{Author contributions:}

DM, ARK, MPM, LD, NS, PG contributed to the conception and design of this study. DM, ARK and PG participated in data collection and contributed to the data analysis. All authors contributed to data interpretation, critically reviewed and revised the manuscript and approved the final submission.

\section{Competing interests:}

Dr. D Michailidou has no disclosures

Dr. AR Khaki: owned stock from Merck and Sanofi within the last 3 years

Dr. MP Morelli has not disclosures

Dr. L Diamantopoulos has no disclosures.

Dr. N Singh has no disclosures.

Dr. P Grivas (all unrelated in the last 3 years):

Consulting: AstraZeneca; Bayer; Bristol-Myers Squibb; Clovis Oncology; Dyania Health, Driver; EMD Serono; Exelixis; Foundation Medicine; Genentech/Roche; Genzyme; GlaxoSmithKline; Heron 
Therapeutics; Immunomedics, Janssen; Merck; Mirati Therapeutics; Pfizer; Seattle Genetics; QED Therapeutics.

Research Funding to Institution: Merck; Pfizer, Clovis Oncology, Bavarian Nordic, Immunomedics, Debiopharm, Bristol-Myers Squibb, QED Therapeutics, GlaxoSmithKline, Kure It Cancer Research.

\section{Funding:}

DM is supported by Pfizer US Pharmaceuticals Group grant, award number 53857367 (funder body did not have any role in this study). ARK is supported by the National Cancer Institute under training grant, award \#T32CA009515. REDCap at ITHS is supported by National Center for Advancing Translational Sciences of National Institutes of Health under Award Number UL1 TR002319. LD acknowledges support from Kure It Cancer Research. NS acknowledges support from Rheumatology Research Foundation and American Heart Association. PG acknowledges support from Seattle Translational Tumor Research Program at Fred Hutchinson Cancer Research Center.

\section{Ethics approval:}

This research was performed under an IRB-approved protocol from the Seattle Cancer Care Alliance IRB (IRB ID: STUDY00008393). All procedures performed complied with the ethical standards of the 1964 Helsinki declaration and its later amendments.

\section{Informed consent:}

Given the retrospective nature of the study, patient consent was waived by the IRB.

\section{References}

1. Kennedy LC, et al. Preexisting autoimmune disease: Implications for immune checkpoint inhibitor therapy in solid tumors. J Natl Compr Canc Netw. 2019 Jun 1; 17(6): 750-757.

2. Postow MA, et al. Immune-Related Adverse Events Associated with Immune Checkpoint Blockade. N Engl J Med 2018;378:158-68.

3. Kumar P, et al. Cancer immunotherapy with checkpoint inhibitor can cause autoimmune adverse events due to loss of Treg homeostasis. Semin Cancer Biol. 2019 Feb 1. pii: S1044-579X(18)301895.

4. Topalian SL, et al. Balance and imbalance in the immune system: life on the edge. Immunity. 2014;41:682-684.

5. Melissaropoulos K, et al. Rheumatic Manifestations in Patients Treated with Immune Checkpoint Inhibitors. Int J Mol Sci. 2020 May; 21(9): 3389. 
6. Cappelli LC, et al. Rheumatic and Musculoskeletal Immune-Related Adverse Events Due to Immune Checkpoint Inhibitors: A Systematic Review of the Literature. Arthritis Care Res (Hoboken). 2017 Nov;69(11):1751-1763.

7. Richter MD, et al. Brief Report: Cancer Immunotherapy in Patients With Preexisting Rheumatic Disease: The Mayo Clinic Experience. Arthritis Rheumatol. 2018 Mar; 70(3):356-360.

8. Haanen $\mathrm{J}$, et al. Autoimmune diseases and immune-checkpoint inhibitors for cancer therapy: review of the literature and personalized risk-based prevention strategy. Ann Oncol. 2020 Mar 17;S09237534(20)-X.

9. Harris PA, et al. Research electronic data capture (REDCap)-a metadata-driven methodology and workflow process for providing translational research informatics support. J Biomed Inform. 2009 Apr;42(2):377-81.

10. Diehl A, et al. Relationships between lymphocyte counts and treatment-related toxicities and clinical responses in patients with solid tumors treated with PD-1 checkpoint inhibitors. Oncotarget. 2017 Dec 14;8(69):114268-114280.

11. Yarchoan M, et al. Targeting neoantigens to augment antitumour immunity. Nat Rev Cancer. 2017; 209-22.

12. Pavan A, et al. Peripheral Blood Markers Identify Risk of Immune-Related Toxicity in Advanced NonSmall Cell Lung Cancer Treated with Immune-Checkpoint Inhibitors. Oncologist. 2019 Aug;24(8):1128-1136.

13. Bagley SJ, et al. Pretreatment neutrophil to lymphocyte ratio as a marker of outcomes in nivolumab treated patients with advanced non-small cell lung cancer. Lung Cancer. 2017 Apr;106:1-7.

14. Peng $L$, et al. Peripheral blood markers predictive of outcome and immune-related adverse events in advanced non-small cell lung cancer treated with PD-1 inhibitor. Cancer Immunol Immunother. 2020 Sep;69(9):1813-1822.

15. Kantola T, et al. Stage-dependent alterations of the serum cytokine pattern in colorectal carcinoma. Br J Cancer. 2012 Nov 6;107(10):1729-36

16. Cheng $\mathrm{H}$, et al. The combination of systemic inflammation-based marker NLR and circulating regulatory $T$ cells predicts the prognosis of resectable pancreatic cancer patients. Pancreatology. 2016 Nov-Dec;16(6):1080-1084.

17. Bilen MA, et al. The prognostic and predictive impact of inflammatory biomarkers in patients who have advanced-stage cancer treated with immunotherapy. Cancer 2019;125:127-134.

18. Franklin RA, et al. The cellular and molecular origin of tumor-associated macrophages. Science 2014;344: 921-5.

19. Pollard JW. Tumour-educated macrophages promote tumour progression and metastasis. Nat Rev Cancer 2004;4:71-8.

20. Martens A, et al. Peripheral Blood Biomarkers Associated with Clinical Outcome of Advanced Melanoma Patients Treated with Ipilimumab. Clin Cancer Res. 2016 Jun 15;22(12):2908-18. 
21. Hemminki K, et al. Familial Associations of Rheumatoid Arthritis With Autoimmune Diseases and Related Conditions. Arthritis Rheum. 2009 Mar;60(3):661-8.

22. Anaya JM, et al. Autoimmune disease aggregation in families with primary Sjo"gren's syndrome. $\mathrm{J}$ Rheumatol 2006;33:2227-34.

23. Sundquist $\mathrm{K}$, et al. Concordant and discordant associations between rheumatoid arthritis, systemic lupus erythematosus and ankylosing spondylitis based on all hospitalisations in Sweden between 1973 and 2004. Rheumatology (Oxford) 2008;47:1199-202.

24. Alarcon-Segovia D, et al, on behalf of the Grupo Latinoamericano de Estudio del Lupus Eritematoso (GLADEL). Familial aggregation of systemic lupus erythematosus, rheumatoid arthritis, and other autoimmune diseases in 1,177 lupus patients from the GLADEL cohort. Arthritis Rheum 2005;52:1138-47.

25. Danlos FX, et al. Safety and efficacy of anti-programmed death 1 antibodies in patients with cancer and pre-existing autoimmune or inflammatory disease. Eur J Cancer. 2018 Mar;91:21-29.

26. Kehl KL, et al. Pre-existing autoimmune disease and the risk of immune-related adverse events among patients receiving checkpoint inhibitors for cancer. Cancer Immunol Immunother. 2019 Jun;68(6):917-926.

27. Haanen $\mathrm{J}$, et al. Rechallenge patients with immune checkpoint inhibitors following severe immunerelated adverse events: review of the literature and suggested prophylactic strategy. J Immunother Cancer.2020 Jun;8(1):e000604.

28. Abdel-Wahab N, et al. Use of Immune Checkpoint Inhibitors in the Treatment of Patients With Cancer and Preexisting Autoimmune Disease A Systematic Review. Ann Intern Med. 2018 Jan 16;168(2):121-130.

29. Calabrese L, et al. Checkpoint immunotherapy: good for cancer therapy, bad for rheumatic diseases. Ann Rheum Dis 2017; 76:1e3.

30. Menzies AM, , et al. Anti-PD-1 therapy in patients with advanced melanoma and preexisting autoimmune disorders or major toxicity with ipilimumab. Ann Oncol. 2017;28:368- 376.

31. Johnson DB, et al. Ipilimumab therapy in patients with advanced melanoma and preexisting autoimmune disorders. JAMA Oncol. 2016;2:234-240.

32. Leonardi GC, et al. Safety of programmed death-1 pathway inhibitors among patients with nonsmall-cell lung cancer and preexisting autoimmune disorders. J Clin Oncol. 2018;36:1905-1912.

33. Wolchok JD, et al. Overall Survival with Combined Nivolumab and Ipilimumab in Advanced Melanoma. N Engl J Med. 2017;377:1345-1356.

34. Kennedy LC, et al. Untangling the Multidisciplinary Care Web: Streamlining Care Through an ImmuneRelated Adverse Events (IRAE) Tumor Board. Target Oncol. 2020;15(4):541-548.

\section{Tables}


Table 1

Demographic characteristics

\begin{tabular}{|c|c|}
\hline & $\underline{N}=470$ \\
\hline Age, median (IQR) & $65(56-71)$ \\
\hline \multicolumn{2}{|l|}{ Sex, N (\%) } \\
\hline Male & $275(59)$ \\
\hline Female & $195(41)$ \\
\hline \multicolumn{2}{|l|}{ Race/Ethnicity, N (\%) } \\
\hline Non-Hispanic White & $408(87)$ \\
\hline Hispanic & $14(3)$ \\
\hline Black & $3(1)$ \\
\hline Asian & $35(7)$ \\
\hline Other & $10(2)$ \\
\hline \multicolumn{2}{|l|}{ Smoking history, N (\%)* } \\
\hline Never & $225(48)$ \\
\hline Former & $199(43)$ \\
\hline Active & $44(9)$ \\
\hline \multicolumn{2}{|l|}{ Cancer site, N (\%) } \\
\hline Lung & $130(28)$ \\
\hline Skin & $93(20)$ \\
\hline Genitourinary & $68(14)$ \\
\hline Gastrointestinal & $53(11)$ \\
\hline Sarcoma & $46(10)$ \\
\hline Hematological malignancy & $37(8)$ \\
\hline Head and neck & $25(5)$ \\
\hline Breast & $7(1)$ \\
\hline Other & $11(2)$ \\
\hline
\end{tabular}




\section{ECOG Performance Status, N (\%)*}

0

$163(35)$

1

$220(47)$

2

76 (16)

3

$10(2)$

\section{ICl given, $\mathbf{N}(\%)$}

Anti-PD-1

$315(67)$

Anti-PD-L1

$56(12)$

Anti-CTLA-4

$2(<1)$

Multiple given concomitantly

88 (19)

Multiple given separately

$9(2)$

Other systemic therapy with $\mathrm{ICl}, \mathrm{N}(\%)$

Yes

$142(30)$

No

$328(70)$

\section{Personal hx of $A D, N(\%)$}

Yes

79 (17)

No

Rheumatologic AD, N (\%)

$47(10)$

Rheumatoid arthritis

6

Polymyalgia rheumatica

6

Psoriasis/Psoriatic arthritis

6

Retroperitoneal fibrosis

Sjogren's syndrome

ANCA negative vasculitis

Undifferentiated connective tissue disease 
Hypothyroidism

Inflammatory bowel disease

Atopic dermatitis

Autoimmune thyroiditis

Chronic inflammatory demyelinating polyneuropathy

Common variable immunodeficiency

Interstitial lung disease

Multiple sclerosis

\section{Family hx of AD, $N(\%)$}

Yes

19 (4)

No

451 (96)

Rheumatologic AD, N (\%)

$14(3)$

Rheumatoid arthritis 3

Systemic lupus erythematosus 3

Psoriatic arthritis/psoriasis 2

Gout 4

Myositis 1

Vasculitis 1

Non-rheumatologic AD, N (\%)

Multiple sclerosis

4

Inflammatory bowel disease

1

History of chronic infection, $\mathbf{N}(\%)$

Yes

40 (9)

No

430 (91) 


\section{Pre-ICI Immunosuppression, N (\%)}

Yes

No
$12(3)$

458 (97)

\section{Labs at ICl initiation, median (IQR)}

ANC $\left(x 10^{\wedge} 3 / \mathrm{ul}\right)$

$\operatorname{ALC}\left(x 10^{\wedge} 3 / \mathrm{ul}\right)$

AMC (x10^3/ul)

Platelets $\left(\mathrm{x} 10^{\wedge} 9 / \mathrm{L}\right)$

NLR

MLR

PLR
$4.3(3.3-6.0)$

$1.2(0.8-1.7)$

$0.5(0.4-0.7)$

229 (177-293)

$3.6(2.4-6.5)$

$0.5(0.3-0.7)$

$183(122-280)$

* 2 patients with missing smoking history and 1 patient missing ECOG PS

Abbreviations: IQR, interquartile range; ECOG Performance status, Eastern Cooperative Oncology Group; ICl, immune checkpoint inhibitor; AD, autoimmune disease; hx, history; Anti-PD-1, antiprogrammed cell death 1; Anti- PD-L1, anti-programmed cell death ligand 1; Anti-CTLA-4, anticytotoxic T-lymphocyte antigen 4, ANCA negative vasculitis, anti-neutrophil cytoplasmic antibody negative vasculitis; ANC, absolute neutrophil count; ALC, absolute lymphocyte count; AMC, absolute monocyte count; NLR, neutrophil to lymphocyte ratio; MLR, monocyte to lymphocyte ratio; PLR, platelet to lymphocyte ratio 
Table 2

Summary of rheumatologic and non-rheumatologic irAEs

\begin{tabular}{|c|c|c|}
\hline & Number & $\%$ \\
\hline All irAEs & 212 & 100 \\
\hline Rheumatologic irAEs & 31 & 15 \\
\hline Inflammatory arthritis & 11 & 5 \\
\hline Rheumatoid arthritis & 4 & 2 \\
\hline Psoriatic arthritis & 4 & 2 \\
\hline Polymyalgia rheumatica & 3 & 1.4 \\
\hline Gout & 3 & 1.4 \\
\hline Arthralgias & 3 & 1.4 \\
\hline Mixed connective tissue disease & 1 & 0.5 \\
\hline Spondylitis & 1 & 0.5 \\
\hline Leukocytoclastic vasculitis & 1 & 0.5 \\
\hline Non rheumatologic irAEs & 181 & 85 \\
\hline Skin & 13 & 6 \\
\hline Non specified facial rash & 2 & 1 \\
\hline Urticaria & 1 & 0.5 \\
\hline Pemphigoid bullous & 3 & 1.4 \\
\hline Eczematous dermatitis & 2 & 1 \\
\hline Lichen planus & 2 & 1 \\
\hline Lichen sclerosus & 1 & 0.5 \\
\hline Vitiligo & 2 & 1 \\
\hline Gastrointestinal & 58 & 27 \\
\hline Colitis & 29 & 14 \\
\hline Diarrhea & 5 & 2.3 \\
\hline Hepatitis & 18 & 8.5 \\
\hline Cholestatic liver disease & 1 & 0.5 \\
\hline Cholangitis & 1 & 0.5 \\
\hline Pancreatitis & 4 & 2 \\
\hline
\end{tabular}




\begin{tabular}{|lll|}
\hline Endocrine & $\mathbf{7 2}$ & $\mathbf{3 4}$ \\
\hline Hypothyroidism & 43 & 20 \\
\hline Thyroiditis & 4 & 2 \\
\hline Adrenal insufficiency & 14 & 7 \\
\hline Type I Diabetes mellitus & 2 & 1 \\
\hline Hypophysitis & 8 & 4 \\
\hline Hypogonadism & 1 & 0.5 \\
\hline Pulmonary & $\mathbf{2 5}$ & $\mathbf{1 2}$ \\
\hline Pneumonitis & 25 & 12 \\
\hline Kidney & $\mathbf{5}$ & $\mathbf{2 . 4}$ \\
\hline Interstitial nephritis & 5 & 2.4 \\
\hline Eye & $\mathbf{2}$ & $\mathbf{1}$ \\
\hline Uveitis & 2 & 1 \\
\hline ENT & $\mathbf{1}$ & $\mathbf{0 . 5}$ \\
\hline Laryngitis & $\mathbf{1}$ & 0.5 \\
\hline Nervous system & $\mathbf{2}$ & $\mathbf{1}$ \\
\hline Neurotoxicity & $\mathbf{1}$ & 0.5 \\
\hline Headache & $\mathbf{1}$ & 0.5 \\
\hline Heart & $\mathbf{1}$ & $\mathbf{0 . 5}$ \\
\hline Myocarditis & 1 & 0.5 \\
\hline Heded adverse events \\
\hline Autoimmune hemolytic anemia & 1 & \\
\hline
\end{tabular}


Table 3A

Association of blood count biomarkers as continuous variables with irAEs and OS

\begin{tabular}{|c|c|c|c|c|c|c|}
\hline & $\underline{\mathrm{irAEs}}$ & & & $\underline{\text { os }}$ & & \\
\hline Blood count biomarkers & $\mathrm{aOR} *$ & $95 \% \mathrm{Cl}$ & $\begin{array}{l}\mathrm{p}- \\
\text { value }\end{array}$ & $\mathrm{aHR}^{*}$ & $95 \% \mathrm{Cl}$ & p-value \\
\hline Absolute neutrophil count (ANC) & 1.01 & $\begin{array}{l}0.95- \\
1.08\end{array}$ & 0.73 & 1.01 & $\begin{array}{l}0.97- \\
1.05\end{array}$ & 0.62 \\
\hline $\begin{array}{l}\text { Absolute lymphocyte } \\
\text { count (ALC) }\end{array}$ & 1.44 & $\begin{array}{l}1.06- \\
1.95\end{array}$ & 0.02 & 0.81 & $\begin{array}{l}0.65- \\
1.01\end{array}$ & 0.06 \\
\hline Absolute monocyte count (AMC) & 0.91 & $\begin{array}{l}0.43- \\
1.93\end{array}$ & 0.81 & 2.04 & $\begin{array}{l}1.18- \\
3.50\end{array}$ & 0.01 \\
\hline Platelet count (PLT) & 1.00 & $\begin{array}{l}1.00- \\
1.00\end{array}$ & 0.45 & 1.00 & $1.00-1.00$ & 0.13 \\
\hline $\begin{array}{l}\text { Neutrophil:Lymphocyte ratio } \\
(N L R)\end{array}$ & 0.97 & $\begin{array}{l}0.92- \\
1.02\end{array}$ & 0.23 & 1.01 & $\begin{array}{l}0.99- \\
1.02\end{array}$ & 0.34 \\
\hline $\begin{array}{l}\text { Monocyte:Lymphocyte ratio } \\
(M L R)\end{array}$ & 0.34 & $\begin{array}{l}0.17- \\
0.70\end{array}$ & 0.003 & 1.27 & $\begin{array}{l}1.05- \\
1.54\end{array}$ & 0.02 \\
\hline Platelet:Lymphocyte ratio (PLR) & 1.00 & $\begin{array}{l}1.00- \\
1.00\end{array}$ & 0.24 & 1.00 & $\begin{array}{l}1.00- \\
1.00\end{array}$ & 0.76 \\
\hline $\begin{array}{l}\text { * Adjusted for age, sex, smoking h } \\
\text { ECOG PS, concomitant other syste } \\
\text { personal history of chronic infectic }\end{array}$ & $\begin{array}{l}y, \text { ca } \\
\text { ther }\end{array}$ & $\begin{array}{l}r \text { type } \\
\text { perso }\end{array}$ & $\begin{array}{l}\text { ologic } \\
\text { amily }\end{array}$ & $\begin{array}{l}\text { laligna } \\
\text { ory of }\end{array}$ & $\begin{array}{l}\text { y vs solid tu } \\
\text { toimmune d }\end{array}$ & ase, \\
\hline
\end{tabular}


Page 19/21 
Blood count biomarkers

Absolute neutrophil count (ANC)

$$
\leq 6.5
$$

1.43

$0.80-2.56$

0.23

Absolute lymphocyte

count (ALC)

$>2.6$

4.12

$1.64-10.42$

0.003

Absolute monocyte count (AMC)

$>0.29$

$>145$

Platelet count (PLT)
1.93

$0.91-4.09$

0.09

Neutrophil:Lymphocyte ratio (NLR)

$$
\leq 5.3
$$

2.08

$1.20-3.58$

0.01

Monocyte:Lymphocyte ratio (MLR)

$$
\leq 0.73
$$

$<0.001$

Platelet:Lymphocyte ratio (PLR)

$$
\leq 534
$$

$0.85-23.42$

0.08

* Adjusted for age, sex, smoking history, cancer type (hematological malignancy vs solid tumor), ECOG PS, concomitant other systemic therapy, personal or family history of autoimmune disease, personal history of chronic infection 
Figures

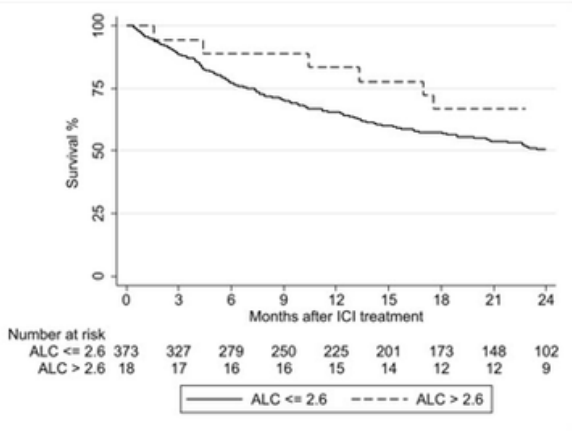

A

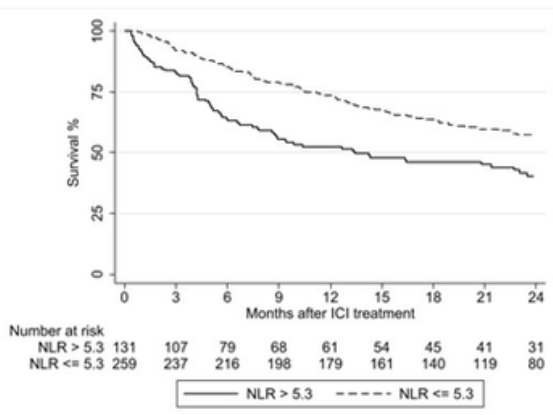

B

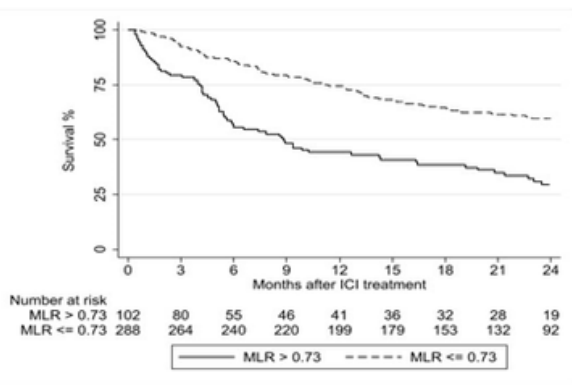

C

\section{Figure 1}

Overall survival (OS) of study population. Kaplan Meier estimates of OS in patients with (A) absolute lymphocyte count $>$ and $\leq 2.6 \mathrm{k} / \mathrm{ul}$, (B) neutrophil to lymphocyte ratio > and $\leq 5.3$ and (C) monocyte to lymphocyte ratio > and $\leq 0.73$. Abbreviations: $\mathrm{HR}$, hazard ratio; ICl, immune checkpoint inhibitors; ALC, absolute lymphocyte count; NLR, neutrophil to lymphocyte ratio; MLR, monocyte to lymphocyte ratio; OS, overall survival; $\mathrm{Cl}$, confidence interval 